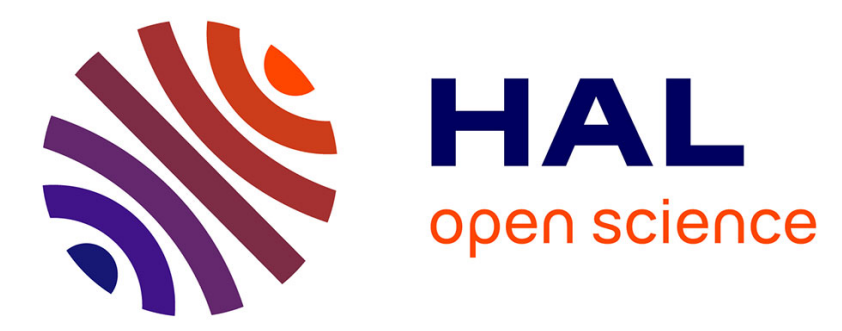

\title{
One-way energy insulation using time-space modulated structures
}

\author{
Kaijun Yi, Sami Karkar, Manuel Collet
}

\section{To cite this version:}

Kaijun Yi, Sami Karkar, Manuel Collet. One-way energy insulation using time-space modulated structures. Journal of Sound and Vibration, 2018, 429, pp.162-175. 10.1016/j.jsv.2018.05.017 . hal02382997

\section{HAL Id: hal-02382997 \\ https://hal.science/hal-02382997}

Submitted on 22 Aug 2021

HAL is a multi-disciplinary open access archive for the deposit and dissemination of scientific research documents, whether they are published or not. The documents may come from teaching and research institutions in France or abroad, or from public or private research centers.
L'archive ouverte pluridisciplinaire HAL, est destinée au dépôt et à la diffusion de documents scientifiques de niveau recherche, publiés ou non, émanant des établissements d'enseignement et de recherche français ou étrangers, des laboratoires publics ou privés. 


\title{
One-way energy insulation using time-space modulated structures
}

\author{
Kaijun Yi ${ }^{\mathrm{a}, 1, *}$, Sami Karkar ${ }^{\mathrm{a}}$, Manuel Collet ${ }^{\mathrm{a}}$ \\ ${ }^{a}$ LTDS UMR5513 Ecole Centrale de Lyon, 36 Avenue Guy de Collongue, 69130 Ecully, France
}

\begin{abstract}
The one-way energy insulation using time-space modulated structures is theoretically studied in this paper. Specifically, the time-space modulated structure in our studies possesses a traveling wave-like modulation. An extended scattering matrix method is developed and verified by comparing to the finite element method. The wave transmission through finite time-space modulated structures is studied using the proposed method. Results show that within the whole stop bands of the two fundamental Bloch modes of time-space modulated structures, one-way wave transmission is achievable. This nonreciprocal phenomenon can be exploited to realize one-way energy isolation in equivalent infinite or semi-infinite systems, which can be found in practice. The one-way energy isolation fails in finite systems due to a frequency conversion phenomenon resulting from the reflection.
\end{abstract}

Keywords: time-space modulation, one-way energy insulation, non-reciprocity, scattering matrix method

\section{Introduction}

Time-space modulated structures are those whose properties are changed both in time and space. The modulation can be either coupled [1] or uncoupled [2] between time and space. In this paper, special attention is payed to the coupled case, which means the modulation acts like a traveling wave in the media. Real time-space modulated structures can be found in many engineering equipments. For example, the axially moving string [3] or rotating ring [4]. They essentially possess space periodicity, the move or rotation further introduces a time periodicity which is coupled with the space one. Also time-space modulated structures can be artificially realized using smart materials like piezoelectric materials, since their effective material properties can be changed by tuning the shunt impedance [5]. This tunable feature has been widely exploited in building metamaterials with tunable band gaps [6-9] and devices with gradient space-varying properties [10-13].

Wave propagation in time-space modulated structures is non-reciprocal. In conventional media, wave motion obeys the reciprocity, which guarantees that if waves can travel from a source to an observer, the opposite propagation path, from the observer to the 
source, is equally possible [14]. This property is violated in time-space modulated structures. The non-reciprocity of them is implied by the symmetry breaking of the dispersion diagram, which has been observed in real axially moving strings [3], rotating rings [4] and structures possessing artificially time-space modulated properties [1, 15-18]. Due to the symmetry breaking of the dispersion diagram, stop bands of the fundamental Bloch modes in time-space modulated structures occupy different frequency ranges $[1,16]$. Unidirectional wave propagation was numerically demonstrated at individual frequencies within these stop bands.

Apart from the above mentioned works, unidirectional wave propagation in time-space modulated structures were also studied by using homogenization methods. K. Lurie [19] designed an array composed of cells with two segments, the properties of these segments are changing in time and space, like the whole array is moving with a constant velocity. He obtained the effective material parameters of the dynamic array and predicted that by appropriately designing the activated array, unidirectional wave propagation can be observed. Following K. Lurie's work, S. Weekes [20] validated his homogenization results by performing direct numerical simulations. L. Shui et al. [21] used an improved multiscale homogenization method to obtain the effective material parameters of the time-space modulated material. According to the homogenization results, they predicted asymmetric wave propagation in the modulated material and numerically demonstrated it.

In brief, the above introduction indicates that the one-way wave propagation is common in diverse time-space modulated structures. This property is desired in achieving one-way energy insulation, which can be used in many engineering applications, such as underwater one-way communication, one-way vibration/noise manipulation, etc. Consequently, time-space modulated structures have drawn and are continuously drawing attention from many researchers in acoustics and mechanics. However, the one-way wave propagation/transmission in them were mainly demonstrated by using numerical methods, such as finite element method [1] and finite-difference time domain method [15], at specific individual frequencies. Limited by the numerical methods, the properties of oneway transmission of acoustic or elastic waves impinging on time-space modulated structures have not been clearly uncovered yet, also the possibility of exploiting time-space modulated structures to realize one-way energy insulation has not been fully discussed.

In this paper, we propose a theoretical method to study the wave transmission through one-dimensional finite time-space modulated structures, and discuss the feasibility of oneway energy isolation. We have the intention of making our works reach out to both acoustical and mechanical engineers. Therefore, the longitudinal motion in slender rods is considered. Our studies can be easily extended to other types of waves, like acoustic waves in air and water, flexural waves in beams, etc. In what follows, time-space modulated structures will be simply called modulated structures. Section 2 studies the dispersion relations and Block modes in modulated rods by using the plane wave expansion (PWE) method. Section 3 proposes a theoretical method to study the reflection and transmission of waves incident on modulated rods. Section 4 presents the results. The proposed theoretical method is verified in section 4.1. The properties of wave transmission are studied in section 4.2. The feasibility of using modulated structures to realize one-way energy insulation is discussed in section 4.3. Finally, section 5 summaries important conclusions. 


\section{Dispersion relations and Bloch modes in modulated rods}

The rod lying along the $x$ axis in figure 1(a) is studied. The density of the rod $\rho_{0}$ is constant and homogeneous, while the Young's modulus is modulated in time and space according to a cosine wave function:

$$
E(x, t)=E_{0}+E_{m} \cos \left(\omega_{m} t-k_{m} x\right)
$$

in which, $E_{0}$ is the Young's modulus when there is no modulation, $E_{m}$ is the modulation amplitude, $\omega_{m}$ and $k_{m}$ are respectively the angular frequency and wavenumber of the modulation wave, whose wavelength is $\lambda_{m}=2 \pi / k_{m}$. The modulation wave propagates along the rod with the speed $v_{m}=\omega_{m} / k_{m}$, as illustrated in figure $1(\mathrm{~b})$.

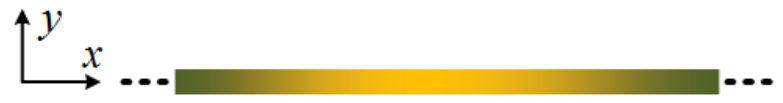

(a)

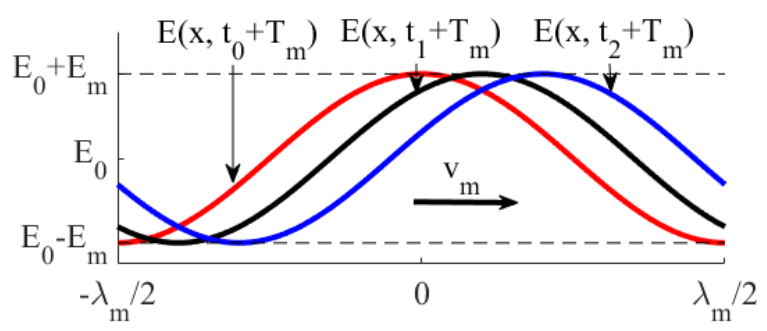

(b)

Figure 1: (a) A slender modulated rod lying along the $x$ axis. (b) The wave-like time-space modulation of the Young's modulus defined by $E(x, t)=E_{0}+E_{m} \cos \left(\omega_{m} t-k_{m} x\right)$. The time period is $T_{m}=2 \pi / \omega_{m}$, the wavelength (space period) is $\lambda_{m}=2 \pi / k_{m}$ and the wave speed is $v_{m}=\omega_{m} / k_{m}$.

In the frequency range where the wavelength is much larger than the diameter of the rod, the longitudinal motion $u(x, t)$ in the modulated rod is governed by:

$$
\frac{\partial}{\partial x}\left[E(x, t) \frac{\partial u(x, t)}{\partial x}\right]-\rho_{0} \frac{\partial^{2} u(x, t)}{\partial t^{2}}=0
$$

To have stable systems, studies in this paper are restricted to the cases satisfying following condition [22]:

$$
\left|\frac{v_{m}}{c_{0}}\right|<\frac{1}{\sqrt{1+\frac{E_{m}}{E_{0}}}}
$$

in which, $c_{0}=\sqrt{E_{o} / \rho_{0}}$ is the phase velocity of the longitudinal wave in an uniform rod.

When the condition in equation (3) is satisfied, the solution of equation (2) can be expressed in the generalized Bloch wave form:

$$
u(x, t)=\sum_{q=-\infty}^{+\infty} U_{q} e^{i\left[\left(\omega+q \omega_{m}\right) t-\left(k+q k_{m}\right) x\right]}
$$


Following the PWE method [1] to obtain the dispersion relations, the Young's modulus function in equation (1) is expanded by using Fourier series:

$$
E(x, t)=\sum_{p=-\infty}^{+\infty} \hat{E}_{p} e^{i p\left(\omega_{m} t-k_{m} x\right)}
$$

in which, $\hat{E}_{p}$ are the Fourier coefficients of corresponding bases.

Substituting equations (4) and (5) into equation (2) and forming the complex inner product with $e^{i n\left(\omega_{m} t-k_{m} x\right)}$ (its complex conjugate appears in the integral), exploiting the orthogonality of the Fourier basis that only the inner products who satisfy $q+p=n$ are nonzero yields:

$$
\sum_{q=-\infty}^{\infty}\left(k+q k_{m}\right)\left(k+n k_{m}\right) \hat{E}_{n-q} U_{q}=\left(\omega+n \omega_{m}\right)^{2} \rho_{0} U_{n}
$$

By truncating the sum in equation (4) to a finite number $2 N+1$ of terms, and evaluating equation (6) for $-N \leq n \leq N$, a well-posed quadratic eigenvalue problem (QEP) is formed with a set of $2 N+1$ equations. The QEP could be solved in terms of $k$ for a given $\omega$ and vice versa. The QEP results in $4 N+2$ eigenvalues and $4 N+2$ corresponding eigenvectors, each of size $2 N+1$. Each eigenvalue together with the corresponding eigenvalue in the form of equation (4) represents a Bloch mode that could exist in the structure. Therefore, there are $4 N+2$ Bloch modes solved from the QEP.

In all the analytical simulations, $\lambda_{m}=0.1 \mathrm{~m}, E_{0}=70 \mathrm{Gpa}, \rho_{0}=2700 \mathrm{~kg} / \mathrm{m}^{3}$ are used. To make our analysis more general, results are discussed using non-dimensional parameters. The dimensionless modulation amplitude $\alpha_{m}=E_{m} / E_{0}$ and dimensionless modulation speed $\beta_{m}=v_{m} / c_{0}$ are used to characterize the modulation wave in equation (1). The dimensionless frequency $\Omega=\lambda_{m} f / c_{0}$ and dimensionless wavenumber $\mu=\lambda_{m} k$ are also used during the discussion of results.

First, the QEP in equation (6) is solved by fixing $k$ and seeking $\omega$. Figures 2(a) and 2(b) show the dispersion relations in a space-only periodic rod and in a time-space modulated rod, respectively. In these figures, shadows indicate the Bragg-type stop bands. In the space-only periodic rod (and also in other conventional spatial periodic media), due to the fold of the dispersion diagram, all Bloch modes that could exist in the rod can be found within the first Brillouin zone (FBZ), where $\mu$ varies in $[-\pi, \pi]$. However, the band folding pattern illustrated in the band diagram of the space-only period rod does not exist in that of the modulated rod, which means that the conventional conception of the FBZ can not be applied to the modulated rod $[1,22]$, i.e., modes in a sufficiently wide wavenumber range must be taken into account.

To study the modes in a wide Brillouin zone, one may need to distinguish and classify the modes, which is difficult to be realized in figure 2. Therefore, in what follows, we use an alternative way to solve the QEP by fixing $\omega$ and seeking $k$. Figure 3 shows the alternative band diagram of the modulated rod with $\alpha_{m}=0.4$ and $\beta_{m}=0.2$. The Bloch modes are classified into positive-going and negative-going groups according to their group velocities, which are calculated by $c_{g}=\partial \omega / \partial k$. Bloch modes in each group are organized according to their wavenumber in an ascending fashion. The $n^{\text {th }}$ Bloch modes 


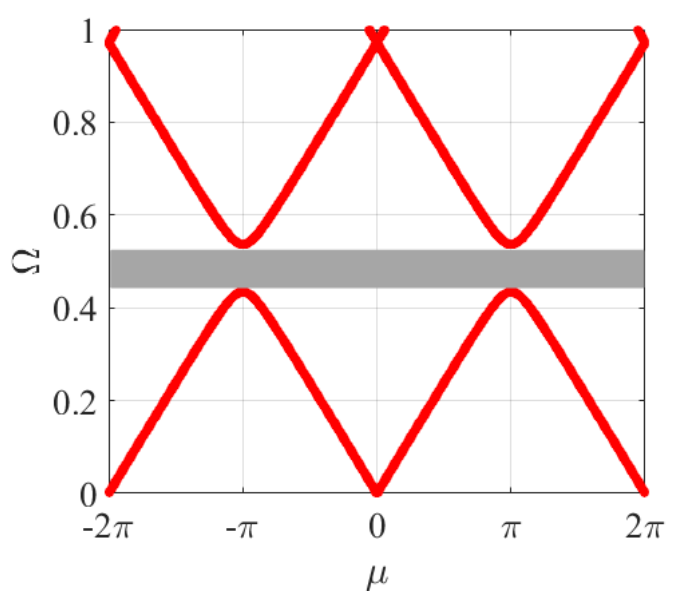

(a) $\alpha_{m}=0.4, \beta_{m}=0$

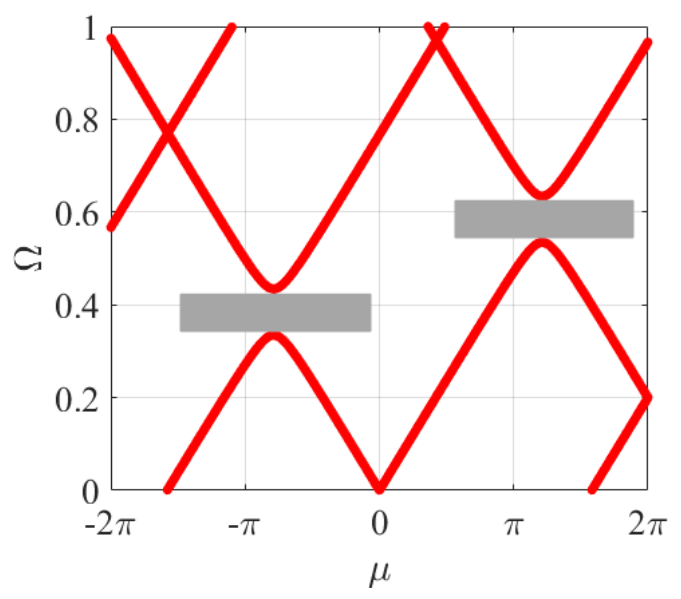

(b) $\alpha_{m}=0.4, \beta_{m}=0.2$

Figure 2: Dispersion relations in a space-only periodic rod with $\alpha_{m}=0.4$ and $\beta_{m}=0$ (figure 2(a)), and in a time-space modulated rod with $\alpha_{m}=0.4$ and $\beta_{m}=0.2$ (figure 2(b)). Shadows indicate the stop bands.

in the positive-going and negative-going groups are respectively represented by $u_{n}^{+}$and $u_{n}^{-}$, with $n=-N, \ldots, 0, \ldots,+N$. According to equation (4), they are expressed as a group of harmonics:

$$
\begin{aligned}
& u_{n}^{+}\left(x, t, k_{n}^{+}, \omega\right)=\sum_{q=-N}^{+N} U_{(n, q)}^{+} e^{i\left[\left(\omega+q \omega_{m}\right) t-\left(k_{n}^{+}+q k_{m}\right) x\right]} \\
& u_{n}^{-}\left(x, t, k_{n}^{-}, \omega\right)=\sum_{q=-N}^{+N} U_{(n, q)}^{-} e^{i\left[\left(\omega+q \omega_{m}\right) t-\left(k_{n}^{-}+q k_{m}\right) x\right]}
\end{aligned}
$$

in which, $k_{n}^{ \pm}$are eigenvalues obtained from the QEP, $\left\{U_{(n,-N)}^{+}, \ldots, U_{(n, 0)}^{+}, \ldots, U_{(n,+N)}^{+}\right\}^{T}$ and $\left\{U_{(n,-N)}^{-}, \ldots, U_{(n, 0)}^{-}, \ldots, U_{(n,+N)}^{-}\right\}^{T}$ are the corresponding eigenvectors.

Figure 3 shows that the band diagram is asymmetric with respect to the $\mu=0$ line. The dispersion curves of positive-going and negative-going modes repeat along an oblique line, whose slope is $2 \pi / \Omega_{m}, \Omega_{m}$ is the dimensionless modulation frequency. For example, when the dispersion curve of $u_{-1}^{+}$mode is horizontally shifted by $\Omega_{m}$, then vertically shifted by $2 \pi$, it totally overlaps the dispersion curve of $u_{0}^{+}$mode. In the figure, the frequency ranges where the real part of $\mu$ is constant are stop bands of corresponding Bloch modes. It can be seen that stop bands of different orders Bloch modes are separated. In some previous papers $[1,16]$, the two stop bands of $u_{0}^{+} / u_{1}^{-}$and $u_{0}^{-} / u_{-1}^{+}$modes in figure 3 are termed directional band gaps, i.e., within these stop bands only positive-going or negative-going modes can propagate. It is remarked here that these descriptions are not appropriate. Because, for example, at frequencies within the stop bands of $u_{0}^{-} / u_{-1}^{+}$modes, even though these two modes can't propagate in the rod, the other orders positive and negative-going Bloch modes can. Therefore, we emphasize again that all these Bloch modes must be taken into account when studying this type of time-space modulated 
systems. Based on this understanding, we developed a method to predict reflection and transmission of waves incident on modulated structures, as will be shown in section 3 .

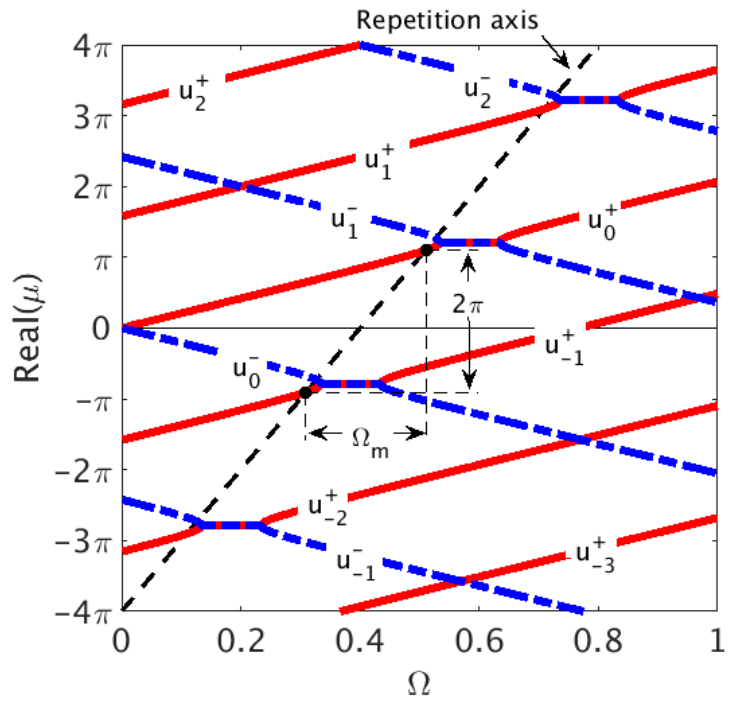

Figure 3: Band diagram of the rod with $\alpha_{m}=0.4, \beta_{m}=0.2 . \Omega_{m}$ is the dimensionless modulation frequency, $u_{n}^{+}$and $u_{n}^{-}$indicate the $n^{\text {th }}$ positive-going and negative-going Bloch modes, respectively. The frequency ranges where the real part of $\mu$ is constant are stop bands of corresponding Bloch modes.

\section{Reflection and transmission of elastic waves incident on modulated rods}

\subsection{Introduction to the scattering matrix method}

Figure 4 illustrates the scattering of incident waves by a one-dimensional scatterer. The scatterer is between the waveguide 1 and 2. $u_{i}^{+}$and $u_{i}^{-}$indicate the incident waves from these two waveguides, respectively. $u_{s}^{-}$and $u_{s}^{+}$indicate the scattered waves. These incident and scattered waves are related by a scattering matrix in the following manner:

$$
\left\{\begin{array}{l}
u_{s}^{-} \\
u_{s}^{+}
\end{array}\right\}=\left[\begin{array}{ll}
R_{11} & T_{21} \\
T_{12} & R_{22}
\end{array}\right]\left\{\begin{array}{l}
u_{i}^{+} \\
u_{i}^{-}
\end{array}\right\}
$$

in which, $R_{11}, T_{12}, T_{21}$ and $R_{22}$ are frequency-dependent coefficients [2].

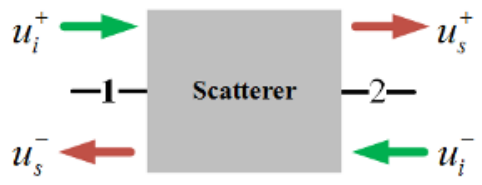

Figure 4: Scattering of incident waves by a one-dimensional scatterer.

The scattering relations in equation (8) can be used to obtain the scattered waves. Also the scattering matrix is very useful to describe the reflection and transmission properties of the scatterer. $R_{11}$ and $T_{12}$ are the reflection and transmission coefficients corresponding 
to the incident wave from the waveguide 1 . On the other hand, $T_{21}$ and $R_{22}$ are the transmission and reflection coefficients corresponding to the incident wave from the waveguide 2. Using these features, the scattering matrix is proposed to judge whether a so-called non-reciprocal device is really non-reciprocal [23, 24].

\subsection{Extending the scattering matrix method to modulated rods}

In this section, the scattering matrix method is extended to study the reflection and transmission of elastic waves incident on time-space modulated structures. The finite modulated rod in figure 5 occupying the region $x_{1} \leq x \leq x_{2}$ is considered, the left and right ends of it are connected to a semi-infinite uniform rod, respectively. The Young's modulus and density of the modulated rod are the same as in section 2, those of the uniform rods are $E_{0}$ and $\rho_{0}$.

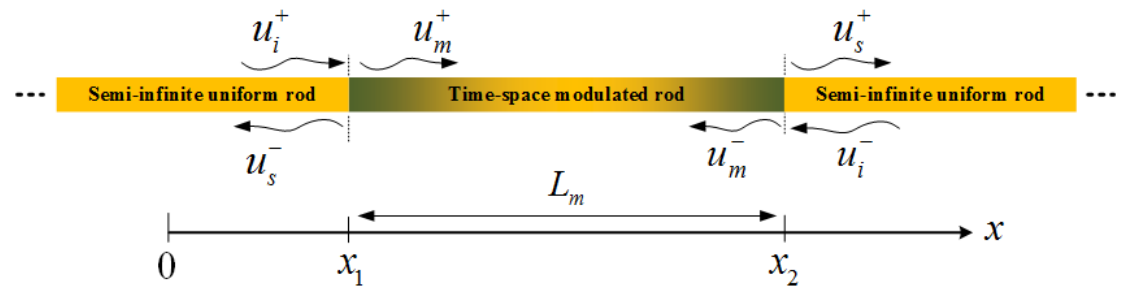

Figure 5: Scattering of incident waves by a modulated rod.

Assume that the following two harmonic waves $u_{i}^{+}$and $u_{i}^{-}$are incident on the modulated rod from left and right sides, respectively:

$$
\begin{aligned}
& u_{i}^{+}(x, t)=A_{0} e^{i \omega\left(t-\frac{x}{c_{0}}\right)} \\
& u_{i}^{-}(x, t)=G_{0} e^{i \omega\left(t+\frac{x}{c_{0}}\right)}
\end{aligned}
$$

in which, $A_{0}$ and $G_{0}$ are corresponding amplitudes, which are known a priori.

Positive-going $\left(u_{m}^{+}\right)$and negative-going $\left(u_{m}^{-}\right)$waves are induced inside the modulated rod. Mathematically these induced waves can be represented as superposition of the Bloch modes supported by the rod. When $2 R+1$ Bloch modes are used, according to equations (7), these waves are represented as:

$$
\begin{aligned}
& u_{m}^{+}(x, t)=\sum_{n=-R}^{+R} C_{n} u_{n}^{+}=\sum_{n=-R}^{+R} \sum_{q=-R}^{+R} C_{n} U_{(n, q)}^{+} e^{i\left[\left(\omega+q \omega_{m}\right) t-\left(k_{n}^{+}+q k_{m}\right) x\right]} \\
& u_{m}^{-}(x, t)=\sum_{n=-R}^{+R} D_{n} u_{n}^{-}=\sum_{n=-R}^{+R} \sum_{q=-R}^{+R} D_{n} U_{(n, q)}^{-} e^{i\left[\left(\omega+q \omega_{m}\right) t-\left(k_{n}^{-}+q k_{m}\right) x\right]}
\end{aligned}
$$

in which, $C_{n}$ and $D_{n}$ are contribution coefficients of corresponding Bloch modes. Note that, the number of Bloch modes used in equations (10) must be smaller than the number of Bloch modes obtained from the QEP, details about this will be discussed later. 
It can be seen from equations (10) that the induced waves inside the modulated rod have harmonic components with frequencies $\omega+q \omega_{m}, q=-R, \ldots, 0, \ldots, R$. Therefore, the scattered waves $\left(u_{s}^{-}\right.$and $\left.u_{s}^{+}\right)$must be superposition of harmonics with all possible frequencies $\omega+q \omega_{m}$ :

$$
\begin{aligned}
& u_{s}^{-}(x, t)=\sum_{q=-R}^{+R} B_{q} e^{i\left(\omega+q \omega_{m}\right)\left(t+\frac{x}{c_{0}}\right)} \\
& u_{s}^{+}(x, t)=\sum_{q=-R}^{+R} F_{q} e^{i\left(\omega+q \omega_{m}\right)\left(t-\frac{x}{c_{0}}\right)}
\end{aligned}
$$

$B_{q}$ and $F_{q}$ are amplitudes of corresponding harmonics.

The incident waves $\left(u_{i}^{+}, u_{i}^{-}\right)$and scattered waves $\left(u_{s}^{-}, u_{s}^{+}\right)$, as well as the induced waves $\left(u_{m}^{+}, u_{m}^{-}\right)$inside the modulated rod must satisfy the continuity of displacement and of force at interfaces $x_{1}, x_{2}$. These conditions are:

$$
\begin{aligned}
u_{i}^{+}\left(x_{1}, t\right)+u_{s}^{-}\left(x_{1}, t\right) & =u_{m}^{+}\left(x_{1}, t\right)+u_{m}^{-}\left(x_{1}, t\right) \\
\left.E_{0} \frac{\partial\left[u_{i}^{+}(x, t)+u_{s}^{-}(x, t)\right]}{\partial x}\right|_{x=x_{1}} & =\left.E\left(x_{1}, t\right) \frac{\partial\left[u_{m}^{+}(x, t)+u_{m}^{-}(x, t)\right]}{\partial x}\right|_{x=x_{1}} \\
u_{m}^{+}\left(x_{2}, t\right)+u_{m}^{-}\left(x_{2}, t\right) & =u_{s}^{+}\left(x_{2}, t\right)+u_{i}^{-}\left(x_{2}, t\right) \\
\left.E\left(x_{2}, t\right) \frac{\partial\left[u_{m}^{+}(x, t)+u_{m}^{-}(x, t)\right]}{\partial x}\right|_{x=x_{2}} & =\left.E_{0} \frac{\partial\left[u_{s}^{+}(x, t)+u_{i}^{-}(x, t)\right]}{\partial x}\right|_{x=x_{2}}
\end{aligned}
$$

Substituting the expressions of waves (equations (9) to (11)) and the two-dimensional Fourier expansion of the Young's modulus of the modulated rod (equation (5)) into the 
continuity conditions results in:

$$
\begin{aligned}
& A_{0} e^{-i \frac{\omega}{c_{0}} x_{1}} e^{i \omega t}+\sum_{q=-R}^{+R}\left\{B_{q} e^{i \frac{\omega+q \omega_{m}}{c_{0}} x_{1}}\right. \\
& \left.-\sum_{n=-R}^{+R}\left[C_{n} U_{(n, q)}^{+} e^{-i\left(k_{n}^{+}+q k_{m}\right) x_{1}}+D_{n} U_{(n, q)}^{-} e^{-i\left(k_{n}^{-}+q k_{m}\right) x_{1}}\right]\right\} e^{i\left(\omega+q \omega_{m}\right) t}=0 \\
& -E_{0} \frac{\omega}{c_{0}} A_{0} e^{-i \frac{\omega}{c_{0}} x_{1}} e^{i \omega t}+\sum_{q=-R}^{+R}\left\{E_{0} \frac{\omega+q \omega_{m}}{c_{0}} B_{q} e^{i \frac{\omega+q \omega m}{c_{0}} x_{1}}\right. \\
& +\sum_{n=-R}^{+R} \sum_{p=-1}^{+1} \hat{E}_{p} e^{-i p k_{m} x_{1}}\left[C_{n}\left(k_{n}^{+}+(q-p) k_{m}\right) U_{(n, q-p)}^{+} e^{-i\left(k_{n}^{+}+(q-p) k_{m}\right) x_{1}}\right. \\
& \left.\left.+D_{n}\left(k_{n}^{-}+(q-p) k_{m}\right) U_{(n, q-p)}^{-} e^{-i\left(k_{n}^{-}+(q-p) k_{m}\right) x_{1}}\right]\right\} e^{i\left(\omega+q \omega_{m}\right) t}=0 \\
& \left\{\sum _ { q = - R } ^ { + R } \left\{\sum_{n=-R}^{+R}\left[C_{n} U_{(n, q)}^{+} e^{-i\left(k_{n}^{+}+q k_{m}\right) x_{2}}+D_{n} U_{(n, q)}^{-} e^{-i\left(k_{n}^{-}+q k_{m}\right) x_{2}}\right]\right.\right. \\
& \left.\left.-F_{q} e^{-i \frac{\omega+q \omega_{m}}{c_{0}} x_{2}}\right\}-G_{0} e^{i \frac{\omega}{c_{0}} x_{2}}\right\} e^{i\left(\omega+q \omega_{m}\right) t}=0 \\
& \left\{\sum _ { q = - R } ^ { + R } \left\{\sum _ { n = - R } ^ { + R } \sum _ { p = - 1 } ^ { + 1 } \hat { E } _ { p } e ^ { - i p k _ { m } x _ { 2 } } \left[C_{n}\left(k_{n}^{+}+(q-p) k_{m}\right) U_{(n, q-p)}^{+} e^{-i\left(k_{n}^{+}+(q-p) k_{m}\right) x_{2}}\right.\right.\right. \\
& \left.+D_{n}\left(k_{n}^{-}+(q-p) k_{m}\right) U_{(n, q-p)}^{-} e^{-i\left(k_{n}^{-}+(q-p) k_{m}\right) x_{2}}\right] \\
& \left.\left.-E_{0} \frac{\omega+q \omega_{m}}{c_{0}} F_{q} e^{-i \frac{\omega+q \omega_{m}}{c_{0}} x_{2}}\right\}+E_{0} \frac{\omega}{c_{0}} G_{0} e^{i \frac{\omega}{c_{0}} x_{2}}\right\} e^{i\left(\omega+q \omega_{m}\right) t}=0
\end{aligned}
$$

The constraint between the truncation order of Bloch modes $R$ introduced in equations (10) and the truncation order $N$ for the QEP is discussed here. When $2 N+1$ positive-going and $2 N+1$ negative-going Bloch modes are obtained from the QEP, the coefficients $U_{(n, q-p)}^{+}$and $U_{(n, q-p)}^{-}$in the second and fourth equations in equations (13) are respectively elements in corresponding eigenvectors $\left\{U_{(n,-N)}^{+}, \ldots, U_{(n, 0)}^{+}, \ldots, U_{(n,+N)}^{+}\right\}^{T}$ and $\left\{U_{(n,-N)}^{-}, \ldots, U_{(n, 0)}^{-}, \ldots, U_{(n,+N)}^{-}\right\}^{T}$. Therefore the integral index $q-p$ must satisfy $-N \leq q-p \leq+N$. For indexes $q$ and $p$, respectively, they satisfy $-R \leq q \leq R$ and $-1 \leq p \leq 1$ (because the Fourier series of the Young's modulus represented in equation (1) only have 3 nonzero terms, namely the terms associated with $\hat{E}_{ \pm 1}$ and $\hat{E}_{0}$ ). Taking all these conditions into account, one can have $R \leq N-1$. Note that the constraint between $R$ and $N$ depends on the type of modulation wave that is used. It is easy to obtain the corresponding constraints for other types of modulation waves following the process introduced here.

By exploiting the orthogonality of harmonic waves $e^{i\left(\omega+q \omega_{m}\right) t}$, the four equations in (13) can be rewritten into the following matrix forms: 


$$
\begin{aligned}
& \mathbf{M}_{B_{1}} \boldsymbol{B}-\mathbf{M}_{C_{1}} \boldsymbol{C}-\mathbf{M}_{D_{1}} \boldsymbol{D}=-A_{0} \mathbf{M}_{A_{1}} \boldsymbol{I}_{0} \\
& \mathbf{M}_{B_{2}} \boldsymbol{B}+\mathbf{M}_{C_{2}} \boldsymbol{C}+\mathbf{M}_{D_{2}} \boldsymbol{D}=A_{0} \mathbf{M}_{A_{2}} \boldsymbol{I}_{0} \\
& \mathbf{M}_{C_{3}} \boldsymbol{C}+\mathbf{M}_{D_{3}} \boldsymbol{D}-\mathbf{M}_{F_{3}} \boldsymbol{F}=G_{0} \mathbf{M}_{G_{3}} \boldsymbol{I}_{0} \\
& \mathbf{M}_{C_{4}} \boldsymbol{C}+\mathbf{M}_{D_{4}} \boldsymbol{D}-\mathbf{M}_{F_{4}} \boldsymbol{F}=-G_{0} \mathbf{M}_{G_{4}} \boldsymbol{I}_{0}
\end{aligned}
$$

here, $\boldsymbol{B}, \boldsymbol{C}, \boldsymbol{D}$ and $\boldsymbol{F}$ are column vectors respectively containing coefficients $B_{q}, C_{n}, D_{n}$ and $F_{q}, n, q=-R, \ldots, 0, \ldots R ; \boldsymbol{I}_{0}$ is a $(2 R+1)$-by- 1 vector, with $\boldsymbol{I}_{0}(R+1)=1$ and other elements are zero; dimensions of matrices represented by $\mathbf{M}_{(.)}$are all $(2 R+1)$-by- $(2 R+1)$, details of them are listed in Appendix A.

By solving equations (14), one can obtain the coefficient vectors $\boldsymbol{B}, \boldsymbol{C}, \boldsymbol{D}$ and $\boldsymbol{F}$, then using the expressions in equations (10), (11), one can obtain the scattered waves, as well as the induced waves inside the modulated part.

To further establish the scattering relations between the incident and scattered waves, coefficients $\boldsymbol{C}$ and $\boldsymbol{D}$ in equations (14) are eliminated and the rest equations are rewritten as:

$$
\left\{\begin{array}{l}
\boldsymbol{B} \\
\boldsymbol{F}
\end{array}\right\}=\left[\begin{array}{ll}
\mathbf{H}_{B F_{11}} & \mathbf{H}_{B F_{12}} \\
\mathbf{H}_{B F_{21}} & \mathbf{H}_{B F_{22}}
\end{array}\right]^{-1}\left[\begin{array}{ll}
\mathbf{H}_{A G_{11}} & \mathbf{H}_{A G_{12}} \\
\mathbf{H}_{A G_{21}} & \mathbf{H}_{A G_{22}}
\end{array}\right]\left\{\begin{array}{l}
A_{0} \\
G_{0}
\end{array}\right\}
$$

Details of the matrices in equations (15) are in Appendix A. Note that, coefficients $A_{0}$ and $G_{0}$ can be complex to include the phase of the incident waves. Therefore, according to equation (15) and the Appendix A, one can find out that the scattering properties of the modulated rod does not depend on the phase of the incident waves.

Equations (15) are the final scattering relations. The corresponding scattering matrix is:

$$
\mathbf{S}=\left[\begin{array}{ll}
\mathbf{H}_{B F_{11}} & \mathbf{H}_{B F_{12}} \\
\mathbf{H}_{B F_{21}} & \mathbf{H}_{B F_{22}}
\end{array}\right]^{-1}\left[\begin{array}{ll}
\mathbf{H}_{A G_{11}} & \mathbf{H}_{A G_{12}} \\
\mathbf{H}_{A G_{21}} & \mathbf{H}_{A G_{22}}
\end{array}\right]
$$

The scattering matrix $\mathbf{S}$ is a $(4 R+2)$-by-2 matrix, as will be shown in section 4 , it is a very handy tool to characterize the reflection and transmission properties of the modulated structures.

\section{Results}

\subsection{Validation of the extended scattering matrix method}

The finite element (FE) method is used as benchmark to verify the extended scattering matrix method developed in section 3.2. Figure 6 shows the rod model used in the FE simulations. It is discretized by two-dimensional Lagrange elements, there are 20 elements per $\lambda_{m}$ length. The left and right parts of the model are uniform rods, the central part is a modulated rod. Absorption boundary conditions (ABC) are used to avoid reflection at the rod ends, therefore to mimic the scattering problem illustrated in figure 5. Since there is a time-varying material parameter in the model, the traditional frequencydomain analysis by performing Fourier transform on the whole governing equations is 
not available. Therefore, in the FE simulations, the time-domain analysis is used. The responses are evaluated by using the generalized $\alpha$ method with a fixed time step equal to $0.0025 \lambda_{m} / c_{0}$. In the theoretical simulations using the extended scattering matrix method, the incident wave is a pure harmonic wave. Therefore, to obtain a similar incident wave in the FE simulations, hanning-windowed tone-burst excitations are used, with 200 cycles.

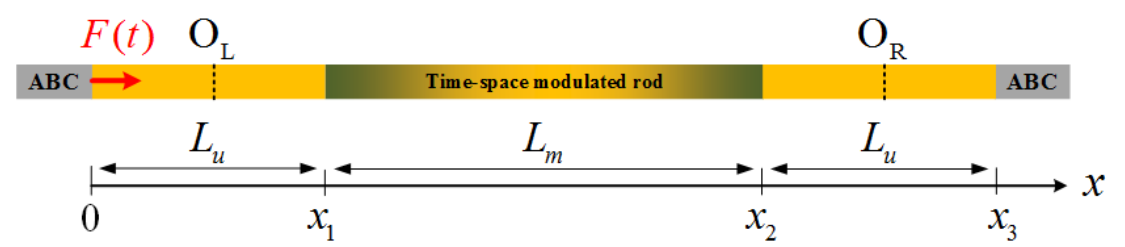

Figure 6: The rod model used in the FE simulations. The left and right parts are uniform rods, the central part is a modulated rod. ABC are applied at the two ends. The modulation parameters of the modulated rod are $\alpha_{m}=0.4, \beta_{m}=0.2$. The lengths are $L_{u}=10 \lambda_{m}$ and $L_{m}=20 \lambda_{m} . O_{L}$ and $O_{R}$ are the two observation points, which are the centers of the two uniform rods, respectively.

In the FE simulations, $\lambda_{m}=0.1 \mathrm{~m}, E_{0}=70 \mathrm{Gpa}, \rho_{0}=2700 \mathrm{~kg} / \mathrm{m}^{3}$. Modulation parameters of the modulated rod are chosen as $\alpha_{m}=0.4, \beta_{m}=0.2$. The lengths of the uniform and modulated parts in figure 6 are $L_{u}=10 \lambda_{m}$ and $L_{m}=20 \lambda_{m}$, respectively. The tone-burst excitation $F(t)$ (with the spectrum centred at $\Omega_{e}$ ) is applied at the left end of the model to generate longitudinal waves incident from the left side of the modulated rod. The responses of the model in time domain are evaluated; then the spectra at two observation points, namely $O_{L}$ and $O_{R}$ in figure 6 , are obtained by performing FFT on the passing waves at these two points. Note that waves at point $O_{L}$ are superposition of the incident and reflected waves, those at point $O_{R}$ are the transmitted waves. These spectra are compared with the theoretical results, which are obtained by solving equations (15) with $G_{0}=0$ at $\Omega_{e}$ (one can also solve equations (14) to have these results). In the theoretical simulations, the modulation parameters and length of the modulated rod are the same to those in the FE simulations. In addition, the truncation order used in the theoretical method is $R=4$. It will be seen that the truncation order $R=4$ is enough to take into account all the significant scattered harmonics. Thus, this truncation order is used in all the following theoretical simulations.

Figures 7 and 8 show the comparison between the numerical and theoretical results at a frequency inside the first stop band of the $u_{0}^{+}$mode $\left(\Omega_{e}=0.584\right)$ and at a frequency outside all the stop bands $\left(\Omega_{e}=0.49\right)$, respectively. The first frequency is the center of the stop band of the $u_{0}^{+}$mode, and the second one is the center between the stop bands of the $u_{0}^{+}$and $u_{0}^{-}$modes (see figure 3). In figures 7 and 8, the black lines represent the results obtained by the FE method. The red matches are the results from the theoretical method. The length of them indicates the amplitude value. To facilitate comparison, all the results are normalized by the maximum amplitude. Recall that $\Omega_{m}=\beta_{m}$ is the dimensionless modulation frequency.

It can be seen that the extended scattering matrix method can accurately predict the reflected and transmitted harmonics. Figures $7(\mathrm{a})$ and $7(\mathrm{~b})$ show discrepancies at some crests. These disagreements are caused by the inconsistency between the incident waves in the theoretical and FE simulations. In particular, as can be seen in figures 7(a) 
and $7(\mathrm{~b})$, when the excitation frequency is $\Omega_{e}=0.584$, most of the incident waves are reflected by the modulated rod (more details about the reflection and transmission will be discussed in section 4.2). These reflected waves will interact with the source in the FE simulation (for example, parts of these waves will be reflected at the source point due to the discontinuity and make contribution to the incident waves), consequently causing the differences between the incident waves in the theoretical and FE simulations. This interpretation is supported by the better agreement shown in figures 8(a) and 8(b), in which case, the reflection from the modulated rod is weak hence the incident waves are less disturbed in the FE simulation.

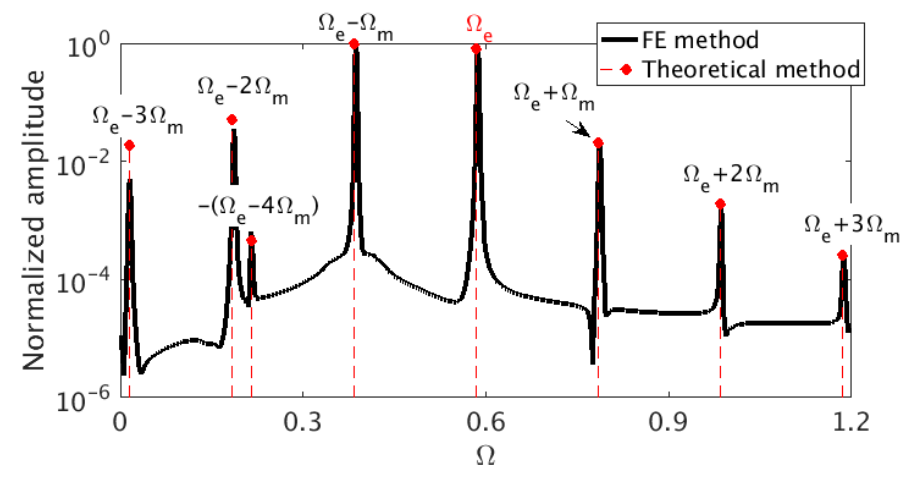

(a) spectra at point $O_{L}$

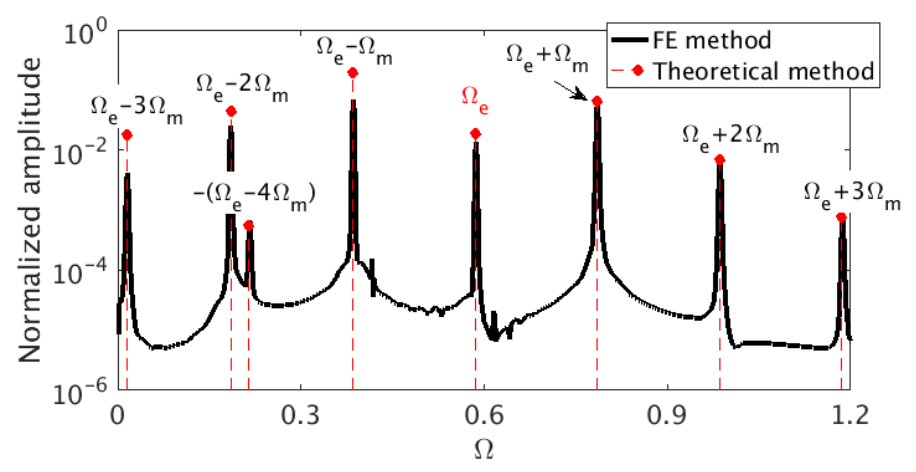

(b) spectra at point $O_{R}$

Figure 7: Comparison between the results obtained by the FE method and the theoretical method when the modulated rod is stimulated by a left incident harmonic wave at frequency $\Omega_{e}=\mathbf{0 . 5 8 4}$. The modulation parameters of the modulated rod are $\alpha_{m}=0.4, \beta_{m}=0.2$. The dimensionless modulation frequency is $\Omega_{m}=0.2$. The length of the modulated rod is $L_{m}=20 \lambda_{m}$.

\subsection{Reflection and transmission properties}

In this section, the reflection and transmission properties within a frequency range covering the first stop bands of the $u_{0}^{+}$and $u_{0}^{-}$modes are studied. The scattering matrix in equation (16) can be divided into four equal quadrants:

$$
\mathbf{S}=\left[\begin{array}{ll}
\boldsymbol{R}_{L} & \boldsymbol{T}_{R} \\
\boldsymbol{T}_{L} & \boldsymbol{R}_{R}
\end{array}\right]
$$




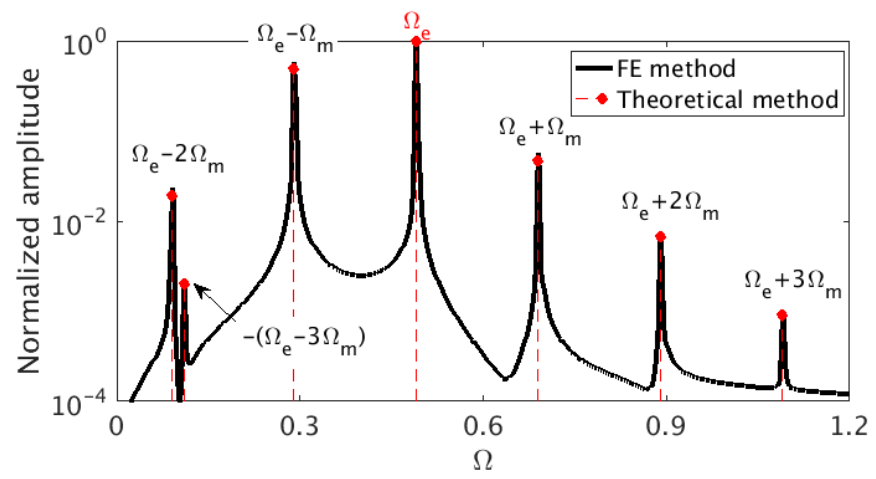

(a) spectra at point $O_{L}$

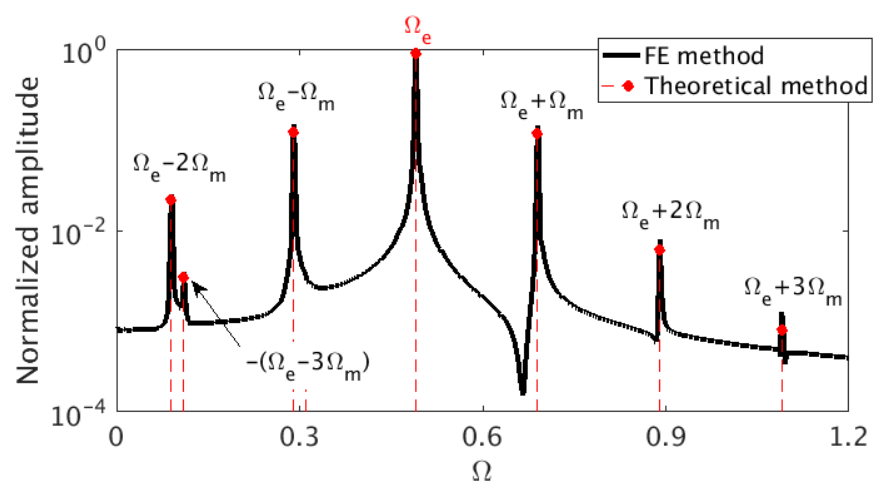

(b) spectra at point $O_{R}$

Figure 8: Comparison between the results obtained by the FE method and the theoretical method when the modulated rod is stimulated by a left incident harmonic wave at frequency $\Omega_{e}=\mathbf{0 . 4 9}$. The modulation parameters of the modulated rod are $\alpha_{m}=0.4, \beta_{m}=0.2$. The dimensionless modulation frequency is $\Omega_{m}=0.2$. The length of the modulated $\operatorname{rod}$ is $L_{m}=20 \lambda_{m}$.

elements in quadrants $\boldsymbol{R}_{L}$ and $\boldsymbol{T}_{L}$ are respectively the reflection and transmission coefficients of a harmonic wave incident from the left side. Elements in quadrants $\boldsymbol{T}_{R}$ and $\boldsymbol{R}_{R}$ are respectively the transmission and reflection coefficients of a harmonic wave incident from the right side.

Figures 9 and 10 show the reflection and transmission coefficients at different frequencies corresponding to a short modulated $\operatorname{rod}\left(L_{m}=20 \lambda_{m}\right)$ and a long modulated rod $\left(L_{m}=40 \lambda_{m}\right)$, respectively. In these figures, each curve is associated with a harmonic composing the reflected or transmitted waves of frequency $\Omega+q \Omega_{m}$.

From figure 9 we can see that, when a harmonic wave with a frequency inside the stop band of the $u_{0}^{+}$mode is incident from the left side, most of it will be reflected (see figure $9(\mathrm{a}))$. In contrary, when this harmonic wave is incident from the right side, most of it will be transmitted (see figure 9(b)). This unidirectional transmission is also observed at frequencies inside the stop band of the $u_{0}^{-}$mode. In this case, a harmonic wave from the right side will be mostly reflected, but the same harmonic wave from the left side will be mostly transmitted.

Also, some nonlinear phenomena occur during the reflection and transmission. It can be seen that, no matter which direction the mono-harmonic wave is incident from, 


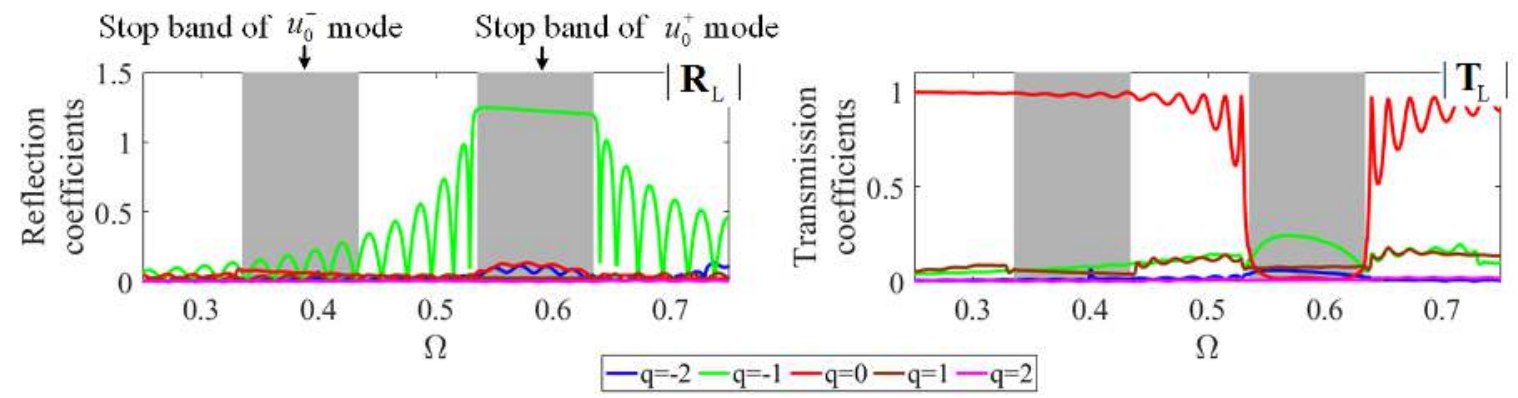

(a) For a harmonic wave incident from the left side.

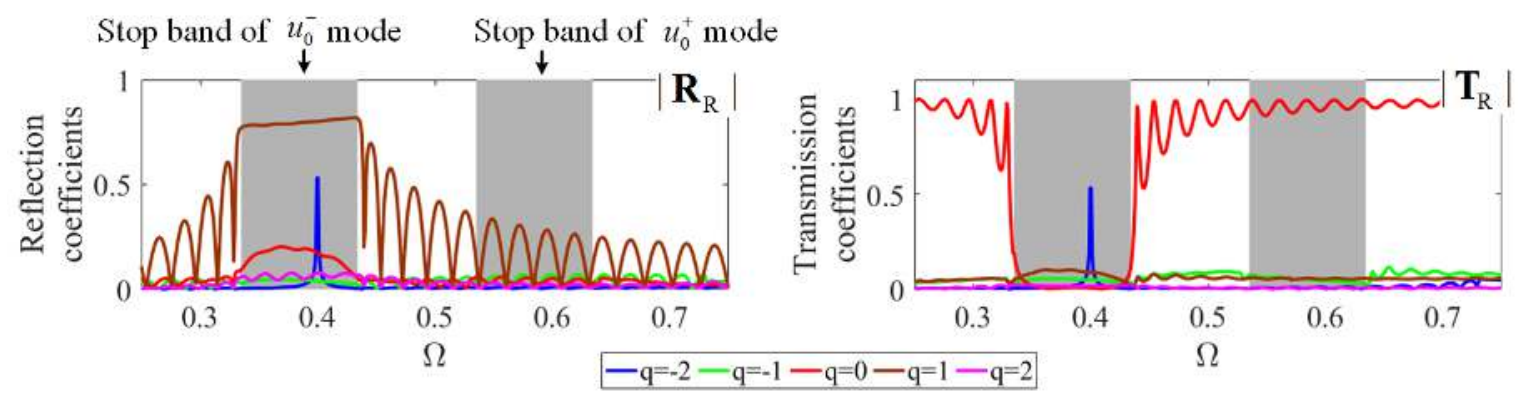

(b) For a harmonic wave incident from the right side.

Figure 9: Reflection and transmission coefficients for harmonic waves incident from two opposite directions when the length of the modulated $\operatorname{rod}$ is $L_{m}=20 \lambda_{m}$. The modulation parameters are $\alpha_{m}=0.4$, $\beta_{m}=0.2$.

the reflected and transmitted waves both contain multiple harmonics with frequencies $\Omega+q \Omega_{m}$, which theoretically verifies the numerical observation in $[18,25]$. Also we can observe frequency conversion from the reflected waves. In particular, the left incident harmonic wave is mainly reflected into the $-1^{\text {st }}$ harmonic with frequency $\Omega-\Omega_{m}$ (see the left figure in figure 9(a)). On the contrary, the right incident harmonic wave is mainly reflected into a harmonic with frequency $\Omega+\Omega_{m}$ (see the left figure in figure $9(\mathrm{~b}))$. The frequency conversion is especially significant in the vicinity of and within the corresponding stop bands since the reflection is more intense at these frequencies. This frequency conversion phenomenon is also numerically demonstrated in $[17,18,26]$ at individual frequencies within the stop bands of the two fundamental modes. The mechanism behind the frequency conversion was explained in the authors' previous paper [27].

It should be noted that obvious exceptional sharp peaks of the amplitude of the $-2^{\text {nd }}$ harmonic at $\Omega=0.4$ are observed. These peaks are caused by the rigid body motion of the rod, because frequency $\left(\Omega-2 \Omega_{m}\right)$ of the $-2^{\text {nd }}$ harmonic tends to 0 as $\Omega$ approaches $\Omega=0.4$. Note that rigid body motion may occur at other frequencies that satisfy $\Omega+q \Omega_{m}=0$. The length of the modulated rod may have significant influences on the reflection and transmission properties of the modulated rod. By comparing the results in figures 9 and 10 we can see that a $20 \lambda_{m}$ long modulated rod is long enough to eliminate those influences in our simulations. 


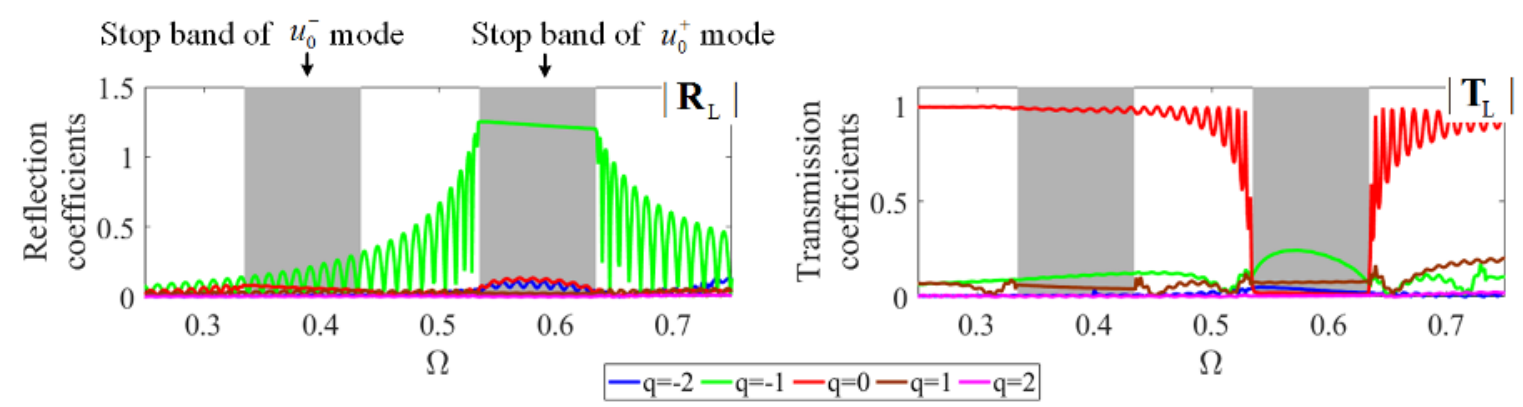

(a) For a harmonic wave incident from the left side.

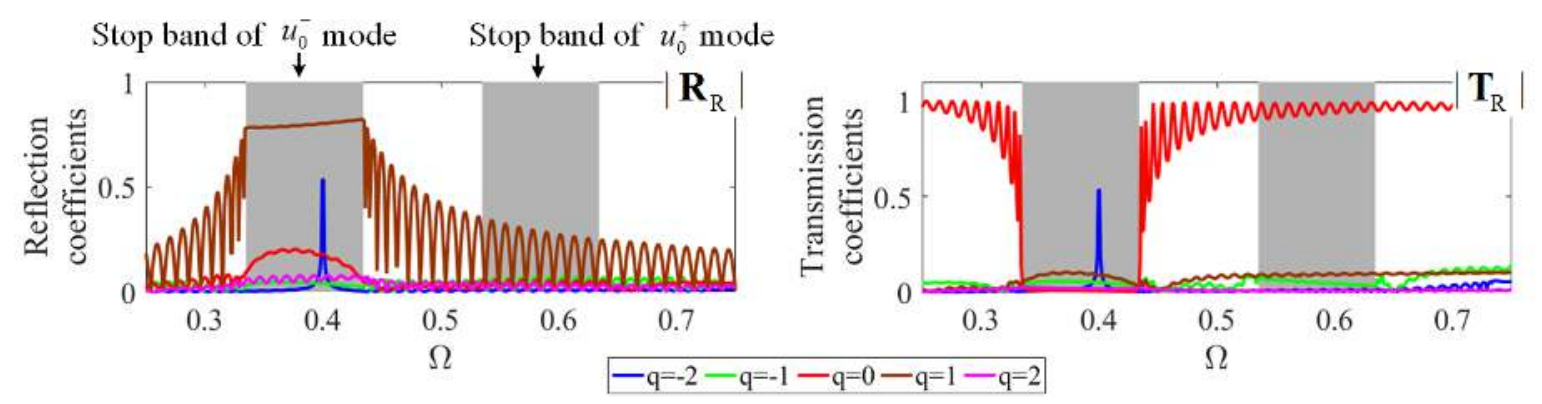

(b) For a harmonic wave incident from the right side.

Figure 10: Reflection and transmission coefficients for harmonic waves incident from two opposite directions when the length of the modulated $\operatorname{rod}$ is $L_{m}=40 \lambda_{m}$. The modulation parameters are $\alpha_{m}=0.4$, $\beta_{m}=0.2$.

\subsection{The feasibility of using modulated structures as one-way energy insulators}

The one-way wave transmission revealed in figures 9 and 10 indicates that, modulated structures can serve as one-way energy insulators in approximate infinite systems or semiinfinite systems. For example, modulated structures could be used to protect coasts and harbors from waves. They will let the offshore disturbance propagates to the ocean however block the impact from the ocean. The ocean can be treated as semi-infinite in these cases. Modulated structures could also be used in finite structures which can be approximated by equivalent infinite ones [28], in these structures the scattered waves are attenuated by damping or radiation therefore are not reflected back to the modulated structures.

However, the one-way energy insulation will fail in finite systems. To demonstrate this, the finite system composed of three parts shown in figure 11 is considered. The central part is a modulated rod, the rest two parts are uniform rods. The left and right ends of the whole system are respectively free and clamped.

The responses of the system excited by a harmonic load $F(t)=L e^{i \omega t}$ applied at the left end (see figure 11) are obtained by using the wave-based theory developed in section 3.2. For the system in figure 11, waves $u_{m}^{+}, u_{m}^{-}, u_{s}^{+}$and $u_{s}^{-}$are still expressed by equations (10) and (11). However, waves $u_{i}^{+}$and $u_{i}^{-}$are unknown now, they should be represented as: 


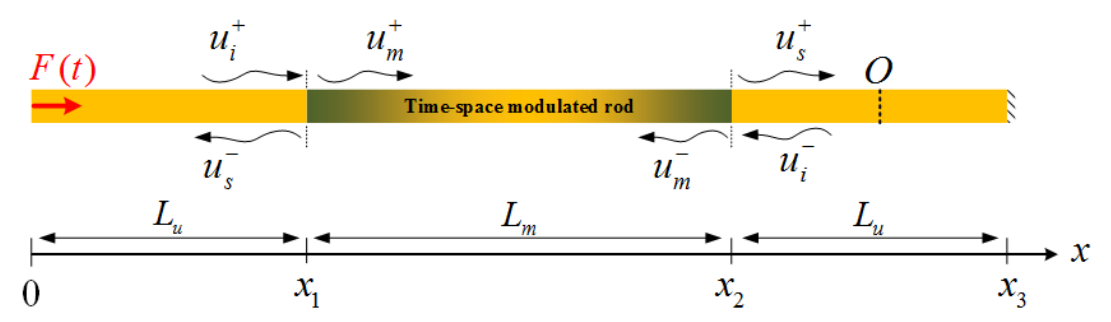

Figure 11: A finite system contains three parts. The left and right parts are uniform rods, the central part is a modulated rod.

$$
\begin{aligned}
& u_{i}^{+}(x, t)=\sum_{q=-R}^{+R} A_{q} e^{i\left(\omega+q \omega_{m}\right)\left(t-\frac{x}{c_{0}}\right)} \\
& u_{i}^{-}(x, t)=\sum_{q=-R}^{+R} G_{q} e^{i\left(\omega+q \omega_{m}\right)\left(t+\frac{x}{c_{0}}\right)}
\end{aligned}
$$

$A_{q}$ and $G_{q}$ are coefficients to be determined.

These waves need to satisfy not only the continuity conditions at $x_{1}$ and $x_{2}$ but also the boundary conditions at the two ends of the system:

$$
\begin{gathered}
\left.E_{0} \frac{\partial\left[u_{i}^{+}(x, t)+u_{s}^{-}(x, t)\right]}{\partial x}\right|_{x=0}=L e^{i \omega t} \\
u_{s}^{+}\left(x_{3}, t\right)+u_{i}^{-}\left(x_{3}, t\right)=0
\end{gathered}
$$

Following the process introduced in section 3.2, a set of linear equations are obtained by first substituting the wave expressions (equations (10), (11) and (18)) and the twodimensional Fourier expansion of the Young's modulus of the modulated rod into the continuity conditions (12) as well as the boundary conditions (19), then exploiting the orthogonality of harmonic functions $e^{i\left(\omega+q \omega_{m}\right) t}$. The unknown coefficients of the waves are solved from these equations. The responses of a particular location in the system are then obtained by superposition of corresponding waves. For example, the responses at point $O$ in figure 11 can be expressed as: $u\left(x_{O}\right)=u_{s}^{+}\left(x_{O}\right)+u_{i}^{-}\left(x_{O}\right)$. In what follows, the responses at this point are studied to verify whether the one-way energy insulation is available in finite systems.

Figure 12 shows the responses at point $O$ in a large frequency band. In the simulations, the lengths are $L_{u}=10 \lambda_{m}$ and $L_{m}=20 \lambda_{m}$. Figure 12(b) shows the frequency responses when the central part is a modulated rod with $\alpha_{m}=0.4, \beta_{m}=0.2$. In this case, the response $u\left(x_{O}\right)$ excited by the excitation at frequency $\Omega$ is composed of multiple harmonics due to the nonlinear phenomena caused by the modulated rod shown in section 4.2. Therefore, the maximum amplitude of the response is evaluated in figure 12(b). As references, figure 12(a) shows the responses when the central part is only space periodic with $\alpha_{m}=0.4, \beta_{m}=0$. The two stop bands of $u_{0}^{-}$and $u_{0}^{+}$modes are totally overlapping in 
this case. In these two figures, results corresponding to two truncation orders are shown. They have very good agreement in both figures, which means that the simulations are convergent.

From figure $12(\mathrm{a})$, it can be seen that within the stop band, the central space-only periodic rod can significantly prevent the energy from being transmitted to the right part. However, when the central rod is time-space modulated, no such effect can be observed within the stop band of $u_{0}^{+}$mode (see figure 12(b)), which verifies that one-way energy insulation fails in finite systems.

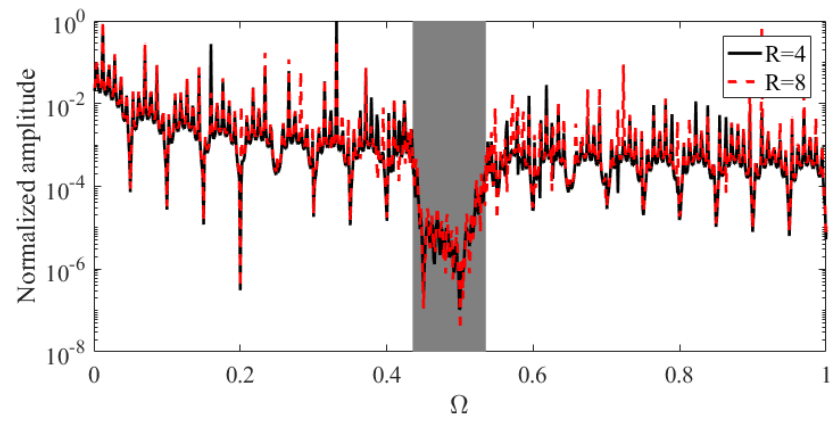

(a)

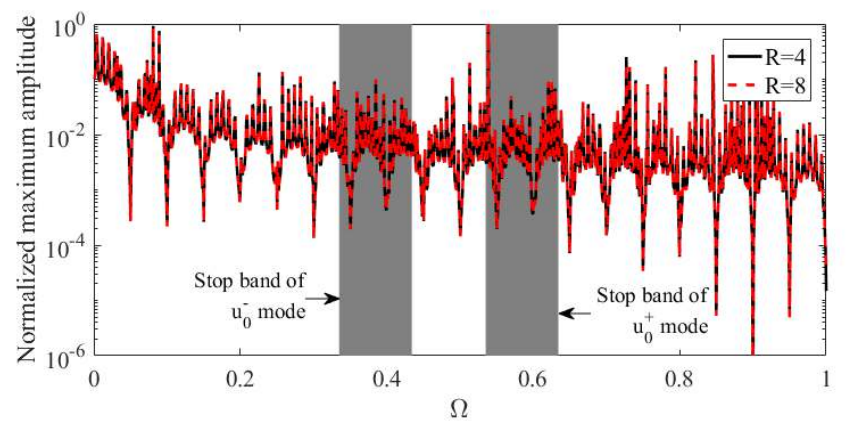

(b)

Figure 12: Frequency responses at point $O$ shown in figure 11: (a) the central part is only space periodic with $\alpha_{m}=0.4, \beta_{m}=0$; (b) the central part is a modulated rod with $\alpha_{m}=0.4, \beta_{m}=0.2$.

The failure of one-way energy insulation in finite systems is mainly caused by the frequency conversion phenomenon. In the finite system shown in figure 11, when a harmonic is incident on the modulated rod from the left side at a frequency $\Omega$ within the stop band of the $u_{0}^{+}$mode, according to the results in figure $9(\mathrm{a})$ or $10(\mathrm{a})$, most of the incident harmonic will be reflected, also the main frequency will be down-converted. Due to the conversion, the main frequency of the reflected waves is outside the stop band of $u_{0}^{+}$mode. Therefore, when this harmonic is reflected back by the left end of the whole system and is incident on the modulated rod the second time, most of it will be transmitted through the modulated rod, causing the failure of the energy insulation. Similar phenomena happen inside the stop band of the $u_{0}^{-}$mode when waves are incident from the right side. 


\section{Conclusions}

This paper theoretically studied the properties of one-way transmission through finite one-dimensional modulated structures and discussed the one-way energy isolation. Caused by the extra time periodicity, modulated structures posses no full band gaps, which are common in space-only periodic media. Stop bands of different orders Bloch modes could be well separated. At any frequency, multiple Bloch modes can be stimulated and make considerable contribution to the forced responses of the modulated structure. Based on this understanding, we developed an extended scattering matrix method to study the reflection and transmission of elastic waves incident on modulated structures. We theoretically demonstrated that, within the whole stop bands of the two fundamental Bloch modes, approximate one-way wave transmission is available. Also it is demonstrated that, when a single harmonic wave is incident on the modulated structure, many frequency components will be generated in the reflected and transmitted waves. Particularly, the main frequency of the reflected waves is up or down converted, depending on the wave incident direction and modulation wave direction. The one-way energy insulation fails in finite systems caused by the frequency conversion. Nevertheless, it can be achieved in those real-life structures which can be approximated by equivalent infinite ones.

\section{Acknowledgement}

Kaijun YI thanks a scholarship provided by the China Scholarship Council to pursue his doctorate in France. 


\section{Appendix A. Details of matrices}

Details of the matrices in equations (14):

$$
\begin{aligned}
& \mathbf{M}_{A_{1}}=\operatorname{diag}\left(e^{-i \frac{\omega+q \omega_{m}}{c_{0}} x_{1}}\right) ; \mathbf{M}_{B_{1}}=\mathbf{M}_{A_{1}}^{-1} \\
& \mathbf{M}_{A_{2}}=\operatorname{diag}\left(E_{0} \frac{\omega+q \omega_{m}}{c_{0}} e^{-i \frac{\omega+q \omega m}{c_{0}} x_{1}}\right) ; \mathbf{M}_{B_{2}}=\mathbf{M}_{B_{1}} \mathbf{M}_{A_{2}} \mathbf{M}_{B_{1}} \\
& \mathbf{M}_{F_{3}}=\operatorname{diag}\left(e^{-i \frac{\omega+q \omega m}{c_{0}} x_{2}}\right) ; \mathbf{M}_{G_{3}}=\mathbf{M}_{F_{3}}^{-1} \\
& \mathbf{M}_{F_{4}}=\operatorname{diag}\left(E_{0} \frac{\omega+q \omega_{m}}{c_{0}} e^{-i \frac{\omega+q \omega_{m}}{c_{0}} x_{2}}\right) ; \mathbf{M}_{G_{4}}=\mathbf{M}_{G_{3}} \mathbf{M}_{F_{4}} \mathbf{M}_{G_{3}} \\
& \mathbf{M}_{C_{1}}(q+R+1, n+R+1)=U_{(n, q)}^{+} e^{-i\left(k_{n}^{+}+q k_{m}\right) x_{1}} \\
& \mathbf{M}_{D_{1}}(q+R+1, n+R+1)=U_{(n, q)}^{-} e^{-i\left(k_{n}^{-}+q k_{m}\right) x_{1}} \\
& \mathbf{M}_{C_{2}}(q+R+1, n+R+1)=\sum_{p=-1}^{+1} \hat{E}_{p} e^{-i p k_{m} x_{1}}\left[\left(k_{n}^{+}+(q-p) k_{m}\right) U_{(n, q-p)}^{+} e^{-i\left(k_{n}^{+}+(q-p) k_{m}\right) x_{1}}\right] \\
& \mathbf{M}_{D_{2}}(q+R+1, n+R+1)=\sum_{p=-1}^{+1} \hat{E}_{p} e^{-i p k_{m} x_{1}}\left[\left(k_{n}^{-}+(q-p) k_{m}\right) U_{(n, q-p)}^{-} e^{-i\left(k_{n}^{-}+(q-p) k_{m}\right) x_{1}}\right] \\
& \mathbf{M}_{C_{3}}(q+R+1, n+R+1)=U_{(n, q)}^{+} e^{-i\left(k_{n}^{+}+q k_{m}\right) x_{2}} \\
& \mathbf{M}_{D_{3}}(q+R+1, n+R+1)=U_{(n, q)}^{-} e^{-i\left(k_{n}^{-}+q k_{m}\right) x_{2}} \\
& \mathbf{M}_{C_{4}}(q+R+1, n+R+1)=\sum_{p=-1}^{+1} \hat{E}_{p} e^{-i p k_{m} x_{2}}\left[\left(k_{n}^{+}+(q-p) k_{m}\right) U_{(n, q-p)}^{+} e^{-i\left(k_{n}^{+}+(q-p) k_{m}\right) x_{2}}\right] \\
& \mathbf{M}_{D_{4}}(q+R+1, n+R+1)=\sum_{p=-1}^{+1} \hat{E}_{p} e^{-i p k_{m} x_{2}\left[\left(k_{n}^{-}+(q-p) k_{m}\right) U_{(n, q-p)}^{-} e^{-i\left(k_{n}^{-}+(q-p) k_{m}\right) x_{2}}\right]}
\end{aligned}
$$

in which, $n, q=-R, \ldots, 0, \ldots R$.

Details of the matrices in equations (15):

$$
\begin{aligned}
& \mathbf{H}_{B F_{11}}=\mathbf{M}_{C_{3}} \mathbf{M}_{C_{1}}^{-1} \mathbf{M}_{B_{1}} \\
& \quad-\left(\mathbf{M}_{C_{3}} \mathbf{M}_{C_{1}}^{-1} \mathbf{M}_{D_{1}}-\mathbf{M}_{D_{3}}\right)\left(\mathbf{M}_{C_{2}} \mathbf{M}_{C_{1}}^{-1} \mathbf{M}_{D_{1}}-\mathbf{M}_{D_{2}}\right)^{-1}\left(\mathbf{M}_{C_{2}} \mathbf{M}_{C_{1}}^{-1} \mathbf{M}_{B_{1}}+\mathbf{M}_{B_{2}}\right) \\
& \mathbf{H}_{B F_{21}}=\mathbf{M}_{C_{4}} \mathbf{M}_{C_{1}}^{-1} \mathbf{M}_{B_{1}} \\
& \quad-\left(\mathbf{M}_{C_{4}} \mathbf{M}_{C_{1}}^{-1} \mathbf{M}_{D_{1}}-\mathbf{M}_{D_{4}}\right)\left(\mathbf{M}_{C_{2}} \mathbf{M}_{C_{1}}^{-1} \mathbf{M}_{D_{1}}-\mathbf{M}_{D_{2}}\right)^{-1}\left(\mathbf{M}_{C_{2}} \mathbf{M}_{C_{1}}^{-1} \mathbf{M}_{B_{1}}+\mathbf{M}_{B_{2}}\right) \\
& \mathbf{H}_{B F_{12}}=-\mathbf{M}_{F_{3}} ; \mathbf{H}_{B F_{22}}=-\mathbf{M}_{F_{4}} \\
& \mathbf{H}_{A G_{11}}=-\mathbf{M}_{C_{3}} \mathbf{M}_{C_{1}}^{-1} \mathbf{M}_{A_{1}} \mathbf{I}_{0} \\
& +\left(\mathbf{M}_{C_{3}} \mathbf{M}_{C_{1}}^{-1} \mathbf{M}_{D_{1}}-\mathbf{M}_{D_{3}}\right)\left(\mathbf{M}_{C_{2}} \mathbf{M}_{C_{1}}^{-1} \mathbf{M}_{D_{1}}-\mathbf{M}_{D_{2}}\right)^{-1}\left(\mathbf{M}_{C_{2}} \mathbf{M}_{C_{1}}^{-1} \mathbf{M}_{A_{1}}-\mathbf{M}_{A_{2}}\right) \boldsymbol{I}_{0} \\
& \mathbf{H}_{A G_{21}}=-\mathbf{M}_{C_{4}} \mathbf{M}_{C_{1}}^{-1} \mathbf{M}_{A_{1}} \boldsymbol{I}_{0} \\
& +\left(\mathbf{M}_{C_{4}} \mathbf{M}_{C_{1}}^{-1} \mathbf{M}_{D_{1}}-\mathbf{M}_{D_{4}}\right)\left(\mathbf{M}_{C_{2}} \mathbf{M}_{C_{1}}^{-1} \mathbf{M}_{D_{1}}-\mathbf{M}_{D_{2}}\right)^{-1}\left(\mathbf{M}_{C_{2}} \mathbf{M}_{C_{1}}^{-1} \mathbf{M}_{A_{1}}-\mathbf{M}_{A_{2}}\right) \boldsymbol{I}_{0} \\
& \mathbf{H}_{A G_{12}}=\mathbf{M}_{G_{3}} \boldsymbol{I}_{0} ; \mathbf{H}_{A G_{22}}=-\mathbf{M}_{G_{4}} \boldsymbol{I}_{0}
\end{aligned}
$$




\section{References}

[1] G. Trainiti and M. Ruzzene. Non-reciprocal elastic wave propagation in spatiotemporal periodic structures. New Journal of Physics, 18(8):083047, 2016.

[2] D. W. Wright and R. S. C. Cobbold. Acoustic wave transmission in time-varying phononic crystals. Smart Materials and Structures, 18(1):015008, 2008.

[3] V. S. Sorokin and J. J. Thomsen. Wave propagation in axially moving periodic strings. Journal of Sound and Vibration, 393:133-144, 2017.

[4] D. Beli, P. B. Silva, and J. R. de França Arruda. Mechanical circulator for elastic waves by using the nonreciprocity of flexible rotating rings. Mechanical Systems and Signal Processing, 98:1077-1096, 2018.

[5] N. W. Hagood and A. V. Flotow. Damping of structural vibrations with piezoelectric materials and passive electrical networks. Journal of Sound and Vibration, 146(2):243-268, 1991.

[6] L. Airoldi and M. Ruzzene. Design of tunable acoustic metamaterials through periodic arrays of resonant shunted piezos. New Journal of Physics, 13(11):113010, 2011.

[7] A. Bergamini, T. Delpero, L. D. Simoni, L. D. Lillo, M. Ruzzene, and P. Ermanni. Phononic crystal with adaptive connectivity. Advanced Materials, 26(9):1343-1347, 2014.

[8] Y. Y. Chen, G. L. Huang, and C. T. Sun. Band gap control in an active elastic metamaterial with negative capacitance piezoelectric shunting. Journal of Vibration and Acoustics, 136(6):061008, 2014.

[9] Y. Fan, M. Collet, M. Ichchou, L. Li, O. Bareille, and Z. Dimitrijevic. A wave-based design of semi-active piezoelectric composites for broadband vibration control. Smart Materials and Structures, 25(5):055032, 2016.

[10] K. Yi, M. Collet, M. Ichchou, and L. Li. Flexural waves focusing through shunted piezoelectric patches. Smart Materials and Structures, 25(7):075007, 2016.

[11] Y. Y. Chen, R. Zhu, M. V. Barnhart, and G. L. Huang. Enhanced flexural wave sensing by adaptive gradient-index metamaterials. Scientific reports, 6, 2016.

[12] K. Yi, M. Collet, S. Chesne, and M. Monteil. Enhancement of elastic wave energy harvesting using adaptive piezo-lens. Mechanical Systems and Signal Processing, 93:255-266, 2017.

[13] K. Yi, M. Monteil, M. Collet, and S. Chesne. Smart metacomposite-based systems for transient elastic wave energy harvesting. Smart Materials and Structures, 26(3):035040, 2017. 
[14] R. Fleury, D. L. Sounas, M. R. Haberman, and A. Alù. Nonreciprocal Acoustics. Acoustics Today, 11(3):14, 2015.

[15] N. Swinteck, S. Matsuo, K. Runge, J. O. Vasseur, P. Lucas, and P. A. Deymier. Bulk elastic waves with unidirectional backscattering-immune topological states in a time-dependent superlattice. Journal of Applied Physics, 118(6):063103, 2015.

[16] H. Nassar, X. C. Xu, A. N. Norris, and G. L. Huang. Modulated phononic crystals: Non-reciprocal wave propagation and willis materials. Journal of the Mechanics and Physics of Solids, 101:10-29, 2017.

[17] H. Nassar, H. Chen, A. N. Norris, M. R. Haberman, and G. L. Huang. Non-reciprocal wave propagation in modulated elastic metamaterials. In Proc. $R$. Soc. A, volume 473, page 20170188. The Royal Society, 2017.

[18] C. Croënne, J. O. Vasseur, O. B. Matar, M. -F. Ponge, P. A. Deymier, A. -C. Hladky-Hennion, and B. Dubus. Brillouin scattering-like effect and non-reciprocal propagation of elastic waves due to spatio-temporal modulation of electrical boundary conditions in piezoelectric media. Applied Physics Letters, 110(6):061901, 2017.

[19] K. A. Lurie. Effective properties of smart elastic laminates and the screening phenomenon. International Journal of Solids and Structures, 34(13):1633-1643, 1997.

[20] S. L. Weekes. Numerical computation of wave propagation in dynamic materials. Applied numerical mathematics, 37(4):417-440, 2001.

[21] L. Shui, Z. Yue, Y. Liu, Q. Liu, J. Guo, and X. He. Novel composites with asymmetrical elastic wave properties. Composites Science and Technology, 113:19-30, 2015.

[22] E. S. Cassedy and A. A. Oliner. Dispersion relations in time-space periodic media: Part istable interactions. Proceedings of the IEEE, 51(10):1342-1359, 1963.

[23] A. A. Maznev, A. G. Every, and O. B. Wright. Reciprocity in reflection and transmission: What is a phonon diode? Wave Motion, 50(4):776-784, 2013.

[24] D. Jalas, A. Petrov, M. Eich, W. Freude, S. Fan, Z. Yu, R. Baets, M. Popovic, A. Melloni, J. D. Joannopoulos, et al. What is-and what is not-an optical isolator. Nature Photonics, 7(8):579, 2013.

[25] J. S. Jensen. Space-time topology optimization for one-dimensional wave propagation. Computer Methods in Applied Mechanics and Engineering, 198(5):705-715, 2009 .

[26] H. Nassar, H. Chen, A. N. Norris, and G. L. Huang. Non-reciprocal flexural wave propagation in a modulated metabeam. Extreme Mechanics Letters, 2017.

[27] K. Yi, M. Collet, and S. Karkar. Frequency conversion induced by time-space modulated media. Phys. Rev. B, 96:104110, Sep 2017. 
[28] H. G. D. Goyder and R. G. White. Vibrational power flow from machines into builtup structures, part i: introduction and approximate analyses of beam and plate-like foundations. Journal of sound and vibration, 68(1):59-75, 1980. 